\title{
Learning Curve as a Knowledge-based Dy- namic Fuzzy Set: A Markov Process Model
}

\author{
Siana Halim ${ }^{1 *}$, Rolly Halim ${ }^{1}$, and Lily Puspa Dewi ${ }^{1}$ \\ ${ }^{1}$ Petra Christian University, Surabaya, Indonesia \\ halimepetra.ac.id
}

\begin{abstract}
In the fuzzy set theory introduced by Zadeh (1965), membership degree of a fuzzy set is determined by a static membership function, i.e., it does not change over time. To improve this condition then Wang introduced the dynamic fuzzy logic. In this concept, the membership degree of a fuzzy set is changing over the time. Intan and Mukaido (2002) introduced the knowledgebased fuzzy set, by means that the membership degree of a set is dependent on the knowledge of a person. Since the knowledge of a person is not static, the knowledge-based fuzzy set can be measured dynamically over time, so that we have the knowledge-based dynamic fuzzy set. In this paper, we approximate the learning process as a knowledge-based dynamic fuzzy set. We consider that the process of learning is dependent on the knowledge of a person from time to time so that we can model the learning process is a Markov process of dynamic knowledge. Additionally, using the triangular fuzzy number, we follow Yabuuchi et al. (2014), for modelling the time difference in the dynamic knowledge fuzzy set as an autoregressive model of order one.
\end{abstract}

Keywords: Learning curve, Fuzzy dynamic, Knowledge-based fuzzy set, Markov process, Autoregressive.

\section{Introduction}

When life is started then the learning process has begun. The life is not only the life of a human being, but also, for example, the life of machines, organization, enterprises, and society. In the learning process, either people or machines, need to do the same task repeatedly in a series of trials and errors. By doing many exercises, knowledge will be learned over time. The learning curve shows the rate of, e.g., a person, a machine, enterprise's, progress in gaining experience or a new skill. It shows the changes over time or processing cost used in gaining experiences.

So far, there exist several mathematical models for representing the learning curve as the function of experience. The common function that is used to figure the general form of all learning curves is the S-curve or the sigmoid function. This curve accu-

adfa, p. 1, 2011.

(C) Springer-Verlag Berlin Heidelberg 2011 
mulates the small steps at first slowly and then gradually it moves faster, as the learning activity mature and reaches its limit. Kucharavy and De Guio (2011), resumed the application of the S-shaped curve, or the sigmoid curve in several types of learning, mainly, in the inventive problem solving, innovation and technology forecasts. Jónás (2007), explained the growth of the learning process in manufacturing and service management. He also extended the concept of reliability, derived from fuzzy theory.

Some learning processes can also represent by exponential functions, especially if the proficiency can increase without limit. Ellis et al. (2016) depicted the student's emerging understanding as exponential growth. In this paper, Ellis et al. exhibited the student's thinking and learning over time to a set of tasks and activities given by the instructors. The exponential function represents most of the students' learning growth since we all hope that their proficiency is increasing without limit. Another way to present the learning phenomena is using the power law function. In this point of view, learning is considered as repetitive tasks, so that the more repetitive has done, the smaller the time needed to learn that task. Tanaka et al. (1986), used the fuzzy regression model for analyzing the learning process. Since the learning process can also be seen as the process of a person or a machine gain knowledge, in this paper, we proposed a dynamic knowledge-based fuzzy set, for representing the learning curve.

\section{Knowledge-based Dynamic Fuzzy Set}

In general, at least there are two types of uncertainty, that is the fuzziness and the randomness (Intan and Mukaido, 2002). The randomness is usually measured using the probabilistic measure, while the fuzziness is measured using membership degrees of elements. In 1965, Zadeh $(1965,1990)$, introduced the fuzzy set. In this set, the membership degrees of elements are represented by a real number in an interval $[0,1]$. The membership degrees of a set is not always the same along the time. People can change their mind, so that they may have the different measurement to the same object from time to time. By these characteristics, then the membership degrees of an object is dynamic.

Researchers in the fuzzy logic, see the dynamic problems from two sides. The first group of researchers (Li, 2008; Silva and Rosano, 2000, Leonid 2012), extended the dynamic terminology in the logic-process sense. While, the second group of researchers, the dynamic in the fuzzy set is represented as the changes of the membership degree as time series (Song and Chissom, 1993; Lee and Chou, 2004; Cheng and Chen, 2018). Intan and Mukaido (2002) stated that the membership degrees of elements in the fuzzy set are dependent on the knowledge of a person. Knowledge is essential for defining a fuzzy set.

Intan et al. (2018) combined the knowledge-based fuzzy set and dynamic fuzzy set to be a knowledge-based dynamic fuzzy set.

Definition 1: let $U=\left\{u_{1}, \ldots, u_{n}\right\}$ be a set of elements and $K=\left\{k_{1}, \ldots, k_{m}\right\}$ be a set of knowledge. A fuzzy set $A$ in $U$ based on a knowledge $k_{i} \in K$ at time $t \in T$, where $T$ is the set of time is notated as $\left.k_{i}(t, A)\right)$. To simplify the notation, the 
$k_{i}(t, A)$ is also defined as the membership function which map $U$ to a closed interval $[0,1]$. This mapping can be written as the membership function of a fuzzy set as follow:

$$
k_{i}(t, A): U \rightarrow[0,1]
$$

A fuzzy set $A$ on $U$ can be written as a fuzzy information table (see Table 1), where $K(t, A)=\left\{k_{1}(t, A), \ldots, k_{m}(t, A)\right\}$ is a knowledge-based dynamic fuzzy time set of $A$

Table 1. Knowledge-based Fuzzy Set of A

\begin{tabular}{|c|c|}
\hline$K(t, A)$ & $u_{1} \ldots u_{n}$ \\
\hline$k_{1}(t, A)$ & $k_{1}(t, A)\left(u_{1}\right) \ldots k_{1}(t, A)\left(u_{n}\right)$ \\
\hline$\vdots$ & $\begin{array}{lll}\vdots & \ddots & \vdots\end{array}$ \\
\hline$k_{m}(t, A)$ & $k_{m}(t, A)\left(u_{1}\right) \ldots k_{m}(t, A)\left(u_{n}\right)$ \\
\hline
\end{tabular}

where $\boldsymbol{k}_{\boldsymbol{i}}(\boldsymbol{t}, \boldsymbol{A})\left(\boldsymbol{u}_{\boldsymbol{j}}\right) \in[\mathbf{0}, \mathbf{1}]$ is the membership degree of element $\boldsymbol{u}_{\boldsymbol{j}}$ in the fuzzy set $\boldsymbol{A}$ which is measured using knowledge $\boldsymbol{k}_{\boldsymbol{i}}$ at time $\boldsymbol{t}$.

\section{Learning curve as a knowledge-based dynamic fuzzy set.}

Supposing knowledge of learners on measuring an object $\mathrm{A}$ at time $\mathrm{t}$ is independent to each other, then we can state that $k_{l}(t, A)$ is independent to $k_{m}(t, A), l \neq m$. However, knowledge of a learner on measuring an object $\mathrm{A}$ at time $t_{i}$ is dependent on the basic knowledge that he or she has, let say $k_{l}\left(t_{0}, A\right)$. Additionally, it is also dependent on the knowledge that he or she gains during the process of learning $k_{l}\left(t_{i-j}, A\right), j=$ $1, \ldots, i$. For simplicity, we will write this learning process as $k\left(t_{i}, A\right), i=1, \ldots, T$. Therefore, in this paper, we propose to define the learning curve as a knowledgebased dynamic fuzzy set. The curve can be represented as the dynamic process of knowledge of a person in measuring an object, especially if the object is not in a crisp set.

\section{$4 \quad$ Knowledge-based dynamic fuzzy set-learning curve as a Markov process}

Since the process of learning is dependent on the knowledge of a person from time to time, we then can assume that the learning process is a Markov process of dynamic knowledge, that is:

$$
k\left(t_{i} \mid t_{i-1}, \ldots, t_{0} ; A\right)=k\left(t_{i} \mid t_{i-1} ; A\right)
$$


The equation (2) can be interpreted as the membership degree given by a person when he or she measures an object $\mathrm{A}$ at time $t_{i}$ given the membership degree based on his or her knowledge on the past is only dependent on the membership degree his or knowledge at a time. In the Markov model (2), we also can say that the person's knowledge on measuring of an object is changing step by step.

The Markov process in this model can be written as the autoregressive model of order one (Kallendberg, 2002). Since the learning process is not a stationary process, but it has the inclination, and settle at particular time, then the series of membership degree of knowledge should be adjusted by taking a difference at order one then, we have

$$
w\left(t_{i} ; A\right)=\Delta k\left(t_{i} ; A\right)=k\left(t_{i} ; A\right)-k\left(t_{i-1} ; A\right)
$$

Now, $w\left(t_{i} ; A\right)$ can be modelled as the autogressive of order one, that is

$$
w\left(t_{i} ; A\right)=\mu+\phi w\left(t_{i-1} ; A\right)+\epsilon
$$

There are several ways to model the fuzzy autoregressive models, Yabuuchi, et al. (2004), used the triangular fuzzy number for constructing the fuzzy autoregressive models. Other models of fuzzy autoregressive were developed by Fukuda and Sunahara (1993). In this study, we follow the Yabuuchi et al. (2004) and defined $w\left(t_{i} ; A\right)$ as a triangular fuzzy number with $w\left(t_{i} ; A\right)=\left(\alpha_{t}, \beta_{t}, \delta_{t}\right), \alpha_{t} \leq \beta_{t} \leq \delta_{t}$

Following the Yabuuchi et al. (2014), the fuzzy autoregressive of order one can be defined as follow:

$$
\begin{gathered}
\widetilde{w}\left(t_{i} ; A\right)=\mu+\phi w\left(t_{i-1} ; A\right)+\epsilon \\
w\left(t_{i} ; A\right) \subseteq \widetilde{w}\left(t_{i} ; A\right), \widetilde{w}\left(t_{i} ; A\right)=\left(\tilde{\alpha}_{t}, \widetilde{\beta}_{t}, \tilde{\delta}_{t}\right)
\end{gathered}
$$

Defined the triangular fuzzy number

$$
u=\left(u_{\alpha}, u_{\beta}, u_{\delta}\right)
$$

To minimize the ambiguity of the model, then:

$$
\begin{array}{cc} 
& \text { Minimize } \sum_{t=2}^{T}\left(\tilde{\delta}_{t}-\tilde{\alpha}_{t}\right) \\
\text { s.t. } & \alpha_{t} \geq \tilde{\alpha}_{t}, \delta_{t} \leq \tilde{\delta}_{t}, t=2, \ldots, T \\
& u_{\alpha} \leq u_{\delta}
\end{array}
$$


and the parameter, $\phi$, can be estimated via Yule Walker estimate (Brockwell and Davis, 1991).

\section{Conclusion}

In this paper, we proposed the knowledge-based dynamic fuzzy set, to represent the learning process. The propose learning curve can be applied to measure the learning process for recognizing objects when a person is not certain about his or her judgment, e.g. "about 10 meters", "about 25 years", "about $36 \mathrm{Kg}$ ", and so on, or if the objects are not in the crisp set, e.g., "smooth", "big", "large", and so on. Additionally, we also model the learning curve as a Markov process and applied the fuzzy autoregressive model of Yabuuchi et al. (2004) for modelling the function of a learning curve. In the future research, we will consider the dependency on the knowledge of other people in the learning process. We also will relax the assumption in the Markov process, so that the dependencies in the autoregressive is not only in order one.

\section{References}

1. Brockwell, P.J., Davis, R.A.: Time Series: Theory and Methods. New York: SpingerVerlag, 238-272, (1991).

2. Cheng, C. H., Chen, C.H.: Fuzzy Time Series Model Based on Weighted Association Rule for Financial Market Forecasting, Expert Systems. Wiley online Library, (2018).

3. Ellis, A. B., Ozgur, Z., Kulow, M., Dogan, M.F., Amidon, J.: An Exponential Growth Learning Trajectory: Student's Emerging Understanding of Exponential Growth through Covariation. Mathematical Thinking and Learning, 18(3), 151-181, (2016).

4. Fukuda, T., Sunahara, Y.: Parameter Identification of Fuzzy Autoregressive Models. IFAC Proceeding, 26(2) Part 5, 689-692, (1993).

5. Intan, R., Mukaidono, M.: On Knowledge-based Fuzzy Sets. International Journal of Fuzzy Systems, 4(2), 655-664, (2002).

6. Jónás, T.: Sigmoid Functions in Reliability based Management. Social and Management Sciences, 15(2), 67-72, (2007).

7. Kallenberg, O.: Foundation of Modern Probability. New-York: Springer-Verlag, (2002).

8. Kucharavy, D., De Guio, R.: Application of S-shaped Curve. Procedia Engineering, 9, 559-572, (2011).

9. Lee, H.S., Chou, M.T.: Fuzzy forecasting based on Fuzzy Time Series. International Journal of Computer Mathematics, 81(7), 781-789, (2004).

10. Leonid, P.: Dynamic Fuzzy Logic, Denotational Mathematics, and Cognition Modeling. Journal of Advanced Mathematics and Application, 1(1), 27-41, (2012).

11. Li, F.: Dynamic Fuzzy Logic and Its Applications. New York: Nova-Science Publisher, (2008).

12. Silva, J. L. P., Rosano, F. L.: Dynamic Fuzzy Logic. Mexican International Conference on Artificial Intelligence (Micai), Lectures Notes in Computer Science, 1793, 661-670, (2000). 
13. Tanaka, H., Shimomura, T., Watada, J.: Identification of Learning Curve based on Fuzzy Regression Model. The Japanese Journal of Ergonomics, 22(5), 253-258, (1986).

14. Yabuuchi, Y., Watada, J., Toyoura, Y.: Fuzzy AR Model of Stock Price, Scientiae Mathematicae Japonicae, 10, 485-492, (2004).

15. Zadeh L. A.: Fuzzy Sets. Information Control, 8, 338-353, (1965).

16. Zadeh, L. A.: Fuzzy sets and Systems. International Journal of General Systems, 17, 129138, (1990). 